\title{
An Experimental Study to Quantify the Rotor-to- Rotor Interaction Characteristics of a Small Unmanned-Aerial-Vehicle
}

\author{
Wenwu Zhou, Zhe Ning, Hui Hu ( $\bowtie)$ \\ ${ }^{1}$ Department of Aerospace Engineering, Iowa State University, Ames, Iowa, 50011, USA \\ (凹) Corresponding author.Email: huhui@iastate.edu
}

\begin{abstract}
The flow interactions between laterally aligned rotors were investigated experimentally to study the rotor-to-rotor interactions on the aerodynamic and aeroacoustic performance of small unmanned aerial vehicles (UAVs). Two identical rotors, similar to the dimensions of Phantom 3 (DJI), were mounted separately on different stages in a wide-open space. High-accuracy force and sound measurements were conducted to document the thrust and noise at various separation distances. The detailed flow structures and corresponding vortex evolutions behind the rotors were resolved clearly by using high-resolution PIV measurements. As the rotor separation distance decreased, intensified flow interactions were noted within the rotors. More specifically, the twinrotor with separation distance of $L=0.05 \mathrm{D}$ exhibited a significantly enhanced thrust fluctuation (i.e., 240\% higher) and augmented noise level (i.e., 3dB) in comparison with that of baseline case. Measured PIV results indicated that a strong recirculation region existed near the top-right of the twin-rotor case, which is believed to be the reason for the increased thrust fluctuations and aeroacoustic noise level.
\end{abstract}

\section{INTRODUCTION}

Recent advances in established control systems and inexpensive electronic devices have greatly encouraged the development of small Unmanned Aerial Vehicles (UAVs) (Bouabdallah et al. 2007; Lucieer et al. 2014; Goebel et al. 2015). Especially for UAVs with rotary-wing system, they are becoming increasingly attractive to engineers due to their unique hovering ability (e.g. vertical take-off and landing) and user-friendly flight controllability. Varieties of applications in civilian fields, such as package delivery, field site surveillance, disease control, video taking, and personal entertainment, are all driving incentives to adopt this new technique. For the sake of providing sufficient thrust, multi-rotor configurations are frequently used in small UAVs during various tasks. For example, the quadrotor, which is one of the most widely-used configurations, can achieve 6 degrees of freedom movement by simply controlling the rotational speeds of individual rotors (Otsuka and Nagatani 2016).

Though UAVs with multi-rotary-wing systems are promising technology for the civilian applications, technical improvements are highly desired for the sake of extending the operating 
time and reducing the aeroacoustic noise. Typically, a representative operating time for small UAVs is less than half an hour, which is far from sufficient for complex tasks. Therefore, boosting the operation time of UAVs is highly desired. One effective method is to improve the aerodynamic performance of multi-rotor UAVs. Since the knowledge pertinent to helicopter rotor dynamics can't be applied to small UAVs directly, it requires comprehensive studies to understand the aerodynamic performance of small UAVs.

Bristeau et al. (Bristeau et al. 2009), who studied the aerodynamic effects of the rotors and their interactions with the UAV body motion, found that the rotor flexibility played an important role in the UAVs' aerodynamics. To obtain first-hand aerodynamic data on commercial UAVs, Russell et al. (Russell et al. 2016) experimentally quantified the thrust coefficients of five multi-rotor UAVs at various wind speeds, rotor speeds, and vehicle attitudes. Similar studies were performed by Brandt and Selig (Brandt and Selig 2011) and Merchant and Miller (Merchant and Miller 2006), who quantified the performance of small propeller at low Reynolds numbers. Aside from the aerodynamic performance, the broadband noise associated with rotor rotating is another issue that needs to be addressed for small UAVs (Gur and Rosen 2009). Aeroacoustic noise not only can be an annoyance to human life, but to the well-being of animals as well(Ditmer et al. 2015). Leslie et al. (Leslie et al. 2008) applied a straight transition strip at the leading edge of blade, and found that the broadband noise of the UAV was greatly reduced. Sinibaldi and Marino (Sinibaldi and Marino 2013) experimentally measured the aeroacoustic signatures of two UAV propellers, and reported that while the optimized propeller was much lower than that of the conventional one at lower thrust, the noise of the two would reach a similar magnitude as the thrust increased to a higher value.

Multi-rotor UAVs commonly feature an even number of rotors that are designed into two sets with either clockwise or counter-clockwise rotations. Very few studies can be found in literature that examined the rotor-to-rotor interactions on the aerodynamic and aeroacoustic performances of UAVs. This is with the exception of Yoon et al.(Yoon et al. 2016), who computationally analyzed the aerodynamics performance of multi-rotor flows in a quadcopter, and found that the rotor interaction had significant effects on the vertical forces in hover motion. Intaratep et al.(Intaratep et al. 2016) measured the acoustic levels of a Phantom II at static thrust conditions and reported a dramatic increase in broadband noise as the rotor number increased from 2 to 4 . Though those studies documented the significant impacts of rotor interactions on the performances of small UAVs, extensive work is still needed to understand the underlying fluid mechanics pertinent to multi-rotor interactions, and to uncover how rotor-to-rotor interactions affect the aerodynamic and aeroacoustic performances in small UAVs.

In the present study, an experimental investigation was performed to evaluate the rotor interactions on the aerodynamic and aeroacoustic performances of small UAVs. Two identical rotors with counter-rotating configuration were mounted separately on two stages and arranged laterally in a wide-open space. The separation distance between rotors was adjusted from $L=$ $0.05 D$ to $L=1.0 D$, which is $0.04 D$ for the DJI Phantom 3. While high-accuracy measurements were conducted to quantify the thrust and noise at various separation distances, a high-resolution PIV system was used to capture the detailed flow structures and the corresponding vortex evolutions behind the rotor. The effects of rotors separation distance (i.e., $L=0.05 D, 0.1 D, 0.2 D$, and $1.0 D$ ) on the aerodynamic and aeroacoustic performances of the UAVs were examined comprehensively based on the measured results. Finally, the resolved flow fields were correlated with the measured results to elucidate the underlying physics to explore/optimize design for next generation multi-rotor drones. 


\section{TEST MODEL AND EXPERIMENTAL SETUPS}

In the present study, the rotor models were made of hard plastic material and manufactured by a rapid prototyping machine (i.e., 3-D printing) that built the rotor models layer-by-layer with a resolution of about 25 microns. As shown schematically in Fig. 1, the rotor has a diameter of 240 $\mathrm{mm}(D=240 \mathrm{~mm})$, which is close to the size of Phantom 3 rotor. An E63 airfoil profile was selected to generate the rotor blade due to its high lift to drag ratio at low Reynolds numbers. The printed rotor was able to provide 3.0 Newton thrust at a rotation speed of 4860 RPM. While the chord length of the rotor blade was set to $11 \mathrm{~mm}$ at the tip, as for other locations (i.e., from tip to $30 \%$ of the blade), they were determined by the optimal chord length equation $C_{r}=\frac{C_{t i p}}{r}$, where $C_{t i p}$ is the chord length at the tip, and $r$ is a non-dimensional radius (i.e., 0.3 at $30 \%$ of the blade and one at the tip). The root section (i.e., from $30 \%$ to $5 \%$ of the blade) is considered as support part, which transits smoothly from the $30 \%$ chord section to the 5\% chord section. It should also be noted that the twist angle of the blade from the tip to $30 \%$ of the blade was adjusted from $11.6^{\circ}$ to $26.3^{\circ}$, and the solidity of the rotor blade was 0.12 in the present study. The rotor models, oriented horizontally, were installed $\sim 300 \mathrm{~mm}$ above the test stage. The experiment stage was $\sim 400 \mathrm{~mm}$ above the ground, therefore it is reasonable to neglect any rotor induced secondary flow effect on the test measurements.

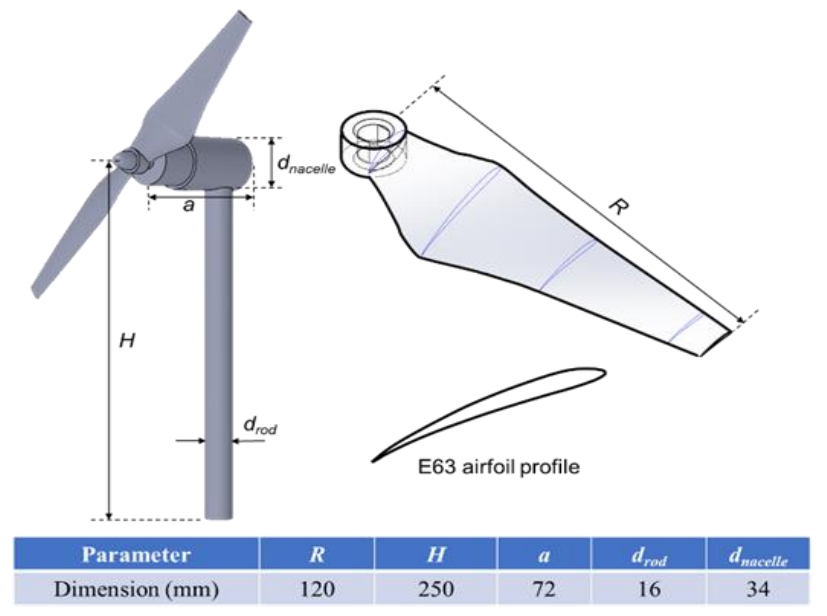

Figure 1. Schematic of rotor model used in the present study.

The experimental study was performed in the Advanced Flow Diagnostic and Experimental Aerodynamic Laboratory located at Iowa State University. Figure 2 (a) shows the experimental setup used for the dynamic thrust and Particle Image Velocimetry (PIV) measurements in the present study, where the main test rig is a custom-built 8020 alumina stage. Equipped with two identical rotors, the propellers in twin-rotor case were rotated in opposite directions (i.e., counterrotating). Their rotational speeds were controlled separately by Electronic Speed Control. The baseline case was simply achieved by removing an identical rotor from the twin-rotor configuration. Comparisons were made between the twin-rotor and baseline cases to quantify the effect of flow interactions on the thrust performance of the UAV rotor. As shown schematically in Fig. 2 (a), a $16 \mathrm{~mm}$ aluminum rod was connected to a high-sensitivity force-moment sensor (JR3 load cell, model 30E12A-I40) to measure the dynamic thrusts of a single rotor. The JR3 load cell, which is composed of foil strain gage bridges, can measure the forces and moments on all three 
orthogonal axes. The precision for force measurements is within $\pm 0.25 \%$ for the full range (40 N). Note that the twin rotors were mounted on two separate stages in order to eliminate the induced mechanical vibration from the nearby rotor. During the experiment, the separation distance $(L)$ between rotor tips was varied from $0.05 D$ to $1.0 D$ to study the rotor interactions on the aerodynamic and aeroacoustic performances of UAV rotors. The JR3 was used to quantify the dynamic load acting on the rotors, which was acquired at a sampling rate of $3000 \mathrm{~Hz}$ for $120 \mathrm{~s}$. The rotor rotational speed was monitored by a Monarch Instrument Tachometer.

As shown schematically in Fig. 2 (b), an aeroacoustic noise measurement was performed in an anechoic chamber. This chamber has a physical dimension of $12 \times 12 \times 9$ feet and its background noise level is lower than $20 \mathrm{~dB}$ with respect to the reference pressure of $20 \mu \mathrm{Pa}$. During the experiment, the microphone was installed $6 D$ away from the center point $O$ of the twin-rotor system. The noise measurement was performed at 5 different angular positions with every $30^{\circ}$ interval, starting from the front position of twin-rotor to $30^{\circ}$ behind the rotors. All noise measurements were performed at the height of rotor hubs in the same plane with laser sheet. Each sound measurement was recorded for $120 \mathrm{~s}$.

In addition, planar PIV measurements were made to quantify the flow interactions between the adjacent rotors, shown in Fig. 2 (a). The air flow was seeded with $\sim 2 \mu \mathrm{m}$ water based droplets generated by fog machine (i.e., ROSCO 1900). Illumination was provided by a double-pulsed Nd:YAG laser (NewWave Gemini 200), adjusted on the second harmonic and emitting two pulses of $200 \mathrm{~mJ}$ with the wavelength of $532 \mathrm{~nm}$ at a repetition rate of $2 \mathrm{~Hz}$. Using a set of high-energy mirrors and optical lenses, the laser beam was shaped into a thin light sheet with a thickness of about $1.0 \mathrm{~mm}$ in the measurement interest. The illuminating laser sheet was firstly aligned horizontally along the induced flow direction, bisecting the hub in the middle of the rotor, to perform planar PIV measurements in the $X-Z$ plane. In the present study, the planer PIV includes both free-run and phase-locked cases. While the free-run PIV were conducted to determine the ensemble-averaged flow statistics behind the rotor, the phase-locked PIV were used to elucidate the dependence of unsteady wake vortices with respect to the position of rotor blades. For the phase-locked PIV measurements, a digital tachometer was used to detect the position of a premarked blade. A pulse signal would be generated by the tachometer as the pre-marked blade passed through the vertical PIV plane. The generated signal was then used as the input signal to Digital Delay Generator (DDG) to trigger the PIV system. Through adjusting the time delays between the input signal from tachometer and the transistor-transistor logic signal from the DDG to trigger the PIV system, different phase angles can be achieved. For each pre-selected phase angle, 300 frames of instantaneous PIV were used to calculate the phase-averaged flow velocity behind the models.

Finally, a stereoscopic PIV (SPIV) experiment was performed to reveal the evolution of vortex structures at different downstream locations behind the rotors (i.e., in $X-Y$ planes). During the experiment, the laser sheet was rotated $90^{\circ}$ from its original position to the vertical direction. Image acquisitions were performed by two 14-bit high-resolution CCD cameras (PCO2000, Cooke Corp.), which were arranged with an angular displacement configuration of about 45 degrees to get a largely overlapped view. With the installation of tilt-axis mounts and the laser illumination plane, the lenses and camera bodies were adjusted to satisfy the Scheimpflug condition. The CCD cameras and double-pulsed Nd:YAG laser were both connected to a Digital Delay Generator (Berkeley Nucleonics, Model 565) to control the timing of the lasers and image acquisitions. A general in-situ calibration procedure was conducted to obtain the mapping functions between the images and object planes for the SPIV measurements. A target plate $\left(\sim 350 \times 350 \mathrm{~mm}^{2}\right)$ with $2 \mathrm{~mm}$ diameter dots spaced at intervals of $8 \mathrm{~mm}$ was used for the in-situ calibration. The mapping 
function used in the present study was a multi-dimensional polynomial function, which is third order for the directions parallel to the laser illumination plane (i.e., $X$ and $Y$ directions), and second order for the direction normal to the laser sheet plane (i.e., $Z$ direction).

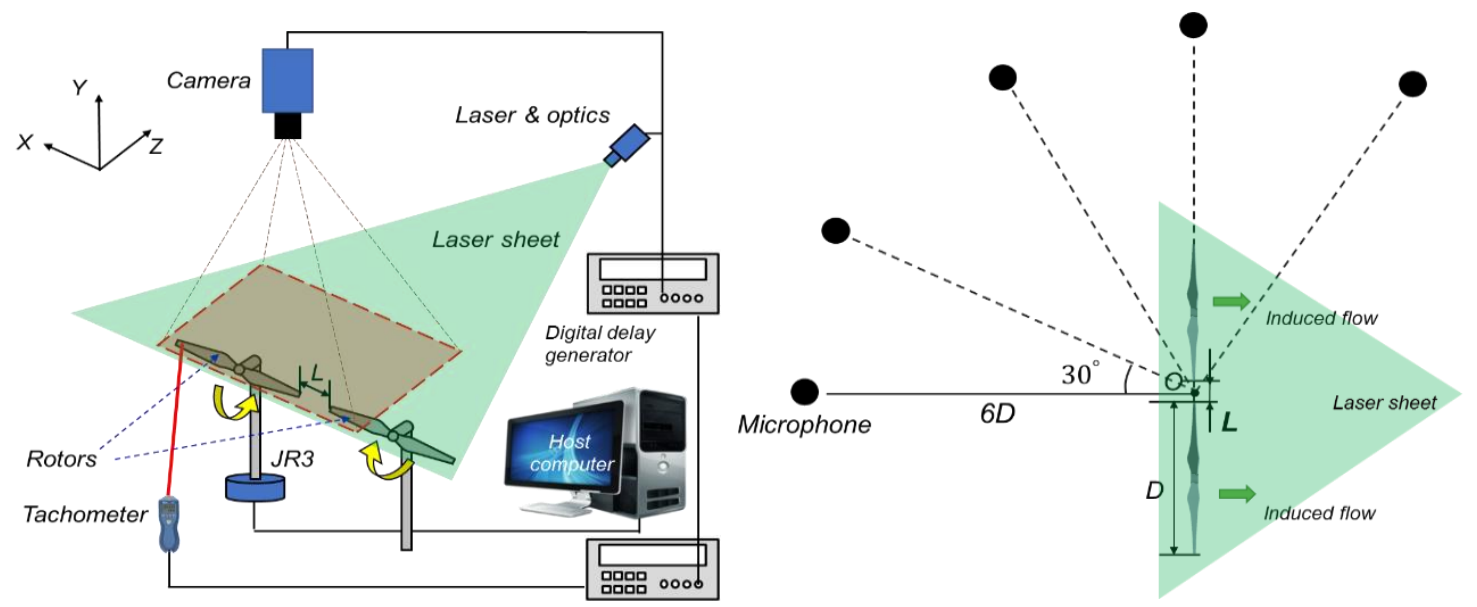

$\begin{array}{ll}\text { (a) Setup for dynamic loads and PIV measurement } & \text { (b) Setup for aeroacoustic measurement }\end{array}$

Figure 2. Experimental setups used in the present study.

In the present study, the instantaneous flow velocity vectors were obtained by using a frameto-frame cross-correlation technique to process the acquired PIV images with an interrogation window size of 32 pixels $\times 32$ pixels. An effective overlap of $50 \%$ of the interrogation windows was employed in planar PIV image processing, which resulted in a spatial resolution of $2.4 \mathrm{~mm}$ (i.e., $0.01 D$ ) for the measurement results. Similar processing methodology was also used for the SPIV image processing. The instantaneous $2 \mathrm{D}$ velocity vectors were then used to reconstruct all three components of the flow velocity vectors in the laser illuminating plane by using the mapping functions obtained through the calibration procedure. After the instantaneous flow velocity vectors $\left(u_{i}, v_{i}, w_{i}\right)$ were determined, the distributions of the ensemble-averaged flow quantities such as mean velocity $(U, V, W)$, normalized turbulence kinetic energy $\left(0.5\left(\overline{u^{\prime 2}}+\overline{v^{2}}+\overline{w^{2}}\right) / U_{\infty}^{2}\right)$, spanwise vorticity $\left(\omega_{y}=\frac{\partial u_{i}}{\partial z}-\frac{\partial w_{i}}{\partial x}\right)$ for the planar PIV measurements, and streamwise vorticity $\left(\omega_{z}=\frac{\partial v_{i}}{\partial x}-\frac{\partial u_{i}}{\partial y}\right)$ for the SPIV measurements were obtained from a sequence of 1,000 instantaneous PIV results (i.e., 300 for phase-locked PIV). The uncertainty level for the PIV measurements is estimated to be within $3 \%$ for the instantaneous velocity vectors, and within $5 \%$ for the measured ensembleaveraged quantities such as vorticity distributions.

\section{MEASUREMENTS RESULTS AND DISCUSSION}

\section{A. Dynamic load measurement results}

Figure 3 presents the normalized thrust coefficient and standard deviation profiles for the twinrotor and baseline cases at various separation distances, where the thrust coefficient (i.e., $\left.C_{T}=\frac{4 T}{\rho n^{2} D^{4} \pi^{3}}\right)$ and standard deviation are normalized by the corresponding quantities of the single rotor, which are 0.013 and 0.21 Newtons, respectively. During the experiment, the rotational speed 
of each rotor (i.e., $n$ ) was kept constant at $81 \mathrm{~Hz}$, but the separation distance between rotor tips was adjusted from $L / D=0.05$ to 1.0. As shown clearly in Fig. 3, though the separation distance was shortened by a factor of 20 , the measured thrust coefficient for the rotor in twin-rotor case was found to drop less than $2 \%$. This suggests that the generated mean thrust is independent of the separation distance. Similar results were also reported by Yoon et al.(Yoon et al. 2016), who computationally studied the aerodynamic interactions of multi-rotor flows. It is worth mentioning that the separation distance also has a negligible effect on the power consumption of rotors. For a fixed rotating frequency, consumed power for the twin-rotor case was approximately same (i.e., difference within $1 \%$ ) for all test cases.

While the separation distance had a limited effect on the thrust coefficient of the rotor, the force fluctuations (i.e., thrust standard deviation) for the monitored rotor in twin-rotor system were found to increase dramatically as the separation distance reduces. More specifically, the measured thrust fluctuations for the $L=0.05 D$ case was found to be $\sim 2.4$ times larger than that of the $L=$ $1.0 D$ case. As mentioned above, since the rotors were mounted on two separated stages, the significant augmentation in force fluctuation for the $L=0.05 \mathrm{D}$ case was believed to be only caused by the intensified flow interactions rather than mechanical vibrations induced by the second rotor. It should also be noted that for $L>0.5 D$, the thrust fluctuation for the twin-rotor case mitigated greatly and eventually reached a similar level with that of the single case. In general, the rotor interaction effect becomes negligible as $L$ larger than $1.0 D$.

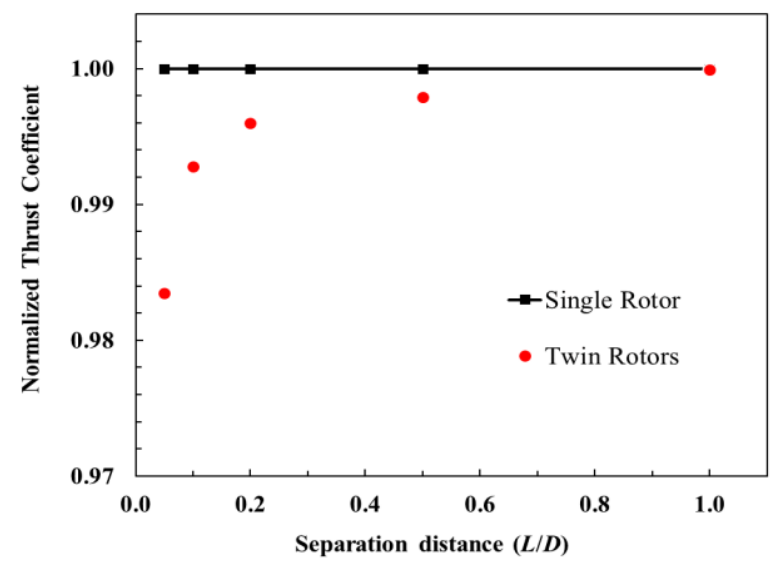

(a). Mean thrust coefficient

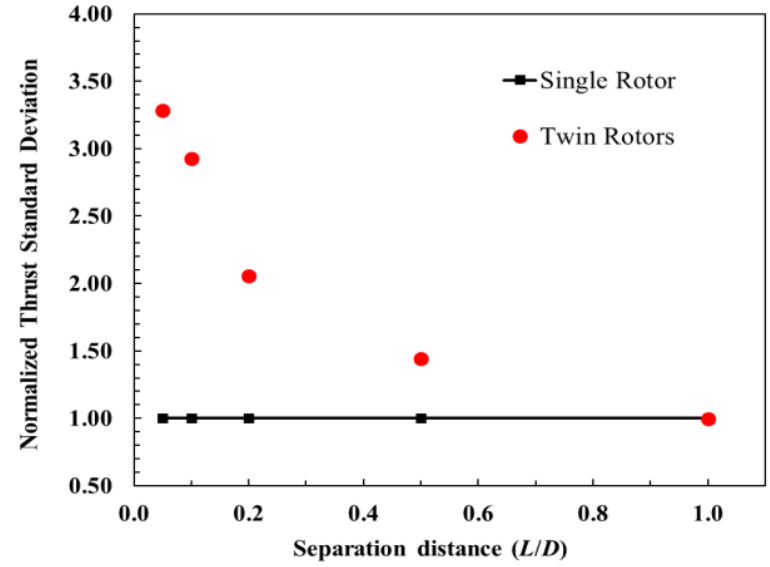

(b). standard deviation of the measured thrust

Figure 3. Effects of separation distance on the mean thrust coefficient (a) and standard deviation (b) of monitored rotor in twin-rotor case, where the thrust coefficient and standard deviation of single rotor (i.e., baseline case) are $0.013 \mathrm{~N}$ and $0.21 \mathrm{~N}$, respectively.

\section{B. Sound measurement results}

The twin-rotor configuration was then investigated to identify the rotor interaction effect on the aeroacoustic performance of small UAVs. The experiment was performed in an anechoic chamber located at Iowa State University. Figure 4 represents the measured sound pressure level (i.e., SPL) distributions at various separation distances (i.e., $L=0.05 D, 0.2 D, 1.0 D$ ). The microphone was installed at five different azimuthal positions (i.e., $\beta=60^{\circ}, 90^{\circ}, 120^{\circ}, 150^{\circ}, 180^{\circ}$ ), which were $6 D$ away from the center and can be approximately treated as far-field locations. Note that, the SPL results displayed in Fig. 4 were the result of the integration of the energy spectrum starting from $20 \mathrm{~Hz}$ to 20,000 Hz. As shown clearly in Fig. 4, the measured SPLs were found to increase 
monotonously as the azimuthal angles increased from $90^{\circ}$ to $180^{\circ}$, reached highest levels at position right ahead of the rotors, while exhibited smallest values by the side of rotor. It is well known that the noise map around a single rotor features a dipole distribution (i.e., symmetric along the rotor plane) (Blackstock 2000). It would reach a maximum noise level at the positions right ahead or behind the rotor (i.e., $\beta=0^{\circ}, 180^{\circ}$ ) but minimum noise at the side positions (i.e., $\beta=90^{\circ}$, $270^{\circ}$ ). As for the twin-rotor configuration, it can be treated as quasi-dipole distribution due to the relatively small gaps within the rotors compared to the microphone locations. It should also be noted that as the separation distance becomes smaller, the measured SPLs would gradually increase. A maximum enhancement of about $3 \mathrm{~dB}$ can be observed for the $L=0.05 D$ case in comparison to the $L=1.0 D$ case at $\beta=180^{\circ}$, which is believed to be caused by the enhanced rotorto-rotor interactions.

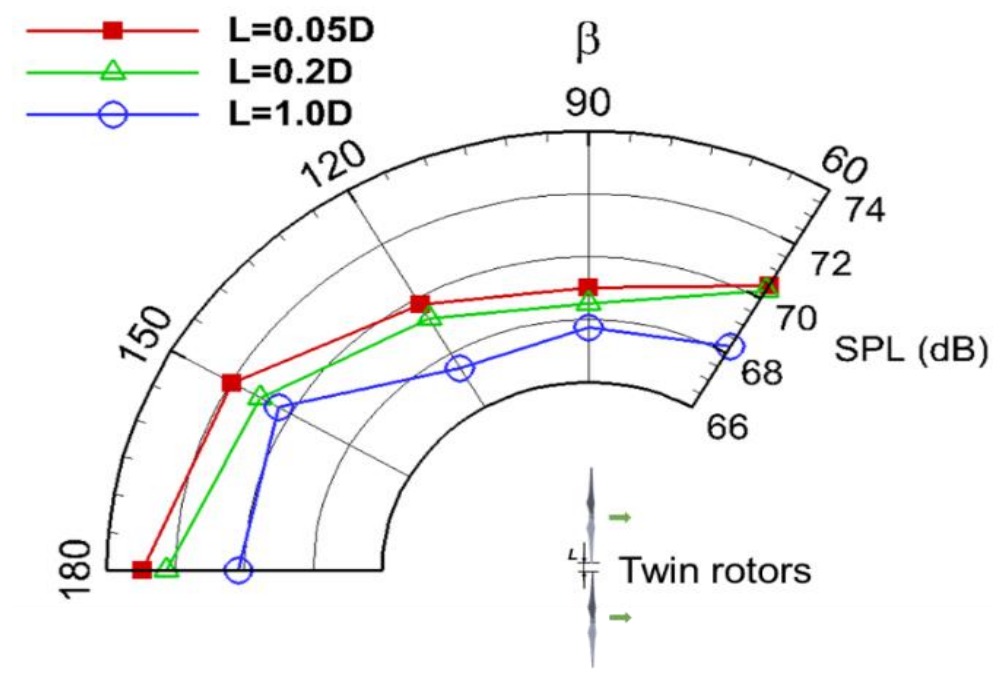

\section{Figure 4. SPL distributions measured at $6 D$ away from the center of the twin-rotor configuration.}

A spectrum analysis was performed to uncover the noise enhancement in terms of tonal noise and broadband noise. Figure 5 shows the measured sound spectrums as a function of blade passing frequency harmonics at azimuthal angles of $\beta=120^{\circ}$ and $180^{\circ}$. Clearly, the measured SPLs for the $L=0.05 \mathrm{D}$ case were found to be much higher than that of the $L=1.0 \mathrm{D}$ case at the integral multiple of the BPF (i.e., BPF, BPF2...), indicating an enhanced tonal noise component around the UAV rotors. For incompressible flow condition, tonal noise is dominated by the loading noise (Blackstock 2000), which is directly associated with the dynamic loading of the rotor blade. Therefore, the augmented tonal noise for the $L=0.05 D$ case is believed to be caused by the intensified thrust fluctuations as mentioned in Fig. 3. It is worth mentioned that the high sparks at $\mathrm{BPF} \approx 20$ were confirmed to be the signal from the electronic motors that used to drive the rotors during the experiment.

Compared to the $L=1.0 \mathrm{D}$ case, the broadband noises for the $L=0.05 \mathrm{D}$ were found to increase mildly at the azimuthal angles of $\beta=120^{\circ}, 180^{\circ}$. Recalling the Lighthill's stress tensor (Blackstock 2000), the broadband noise is largely determined by the complex turbulent flow structures and shear layers, such as secondary flow distortions, blade trailing edge vortices, and tip vortices. As reported by Carolus et al. (Carolus et al. 2007), turbulent kinetic energy (TKE) is important parameters that can be used to evaluate the broadband noise level. Enhanced TKE level will lead 
to augmented broadband noise. Therefore, the broadband noise measurements in the present study suggest an augmented TKE level for the twin-rotor configuration as the separation distance decrease, which was confirmed by the measured PIV results shown below.

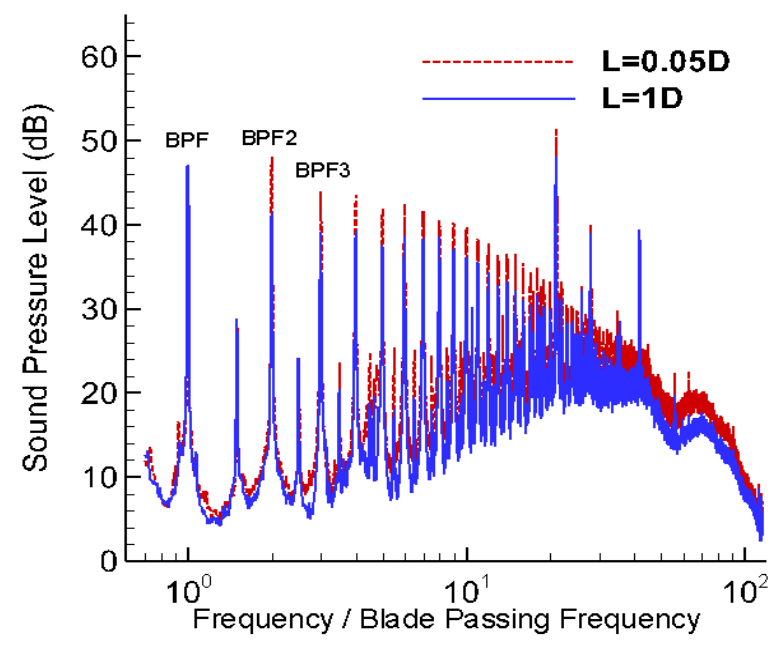

(a) $\beta=120^{\circ}$

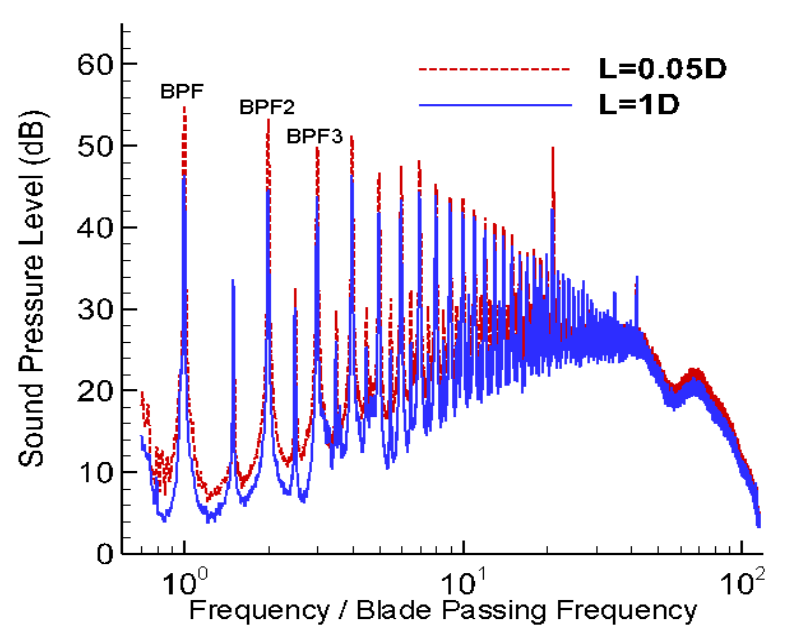

(b) $\beta=180^{\circ}$

Figure 5. Comparison of the measured sound spectrums between the $L=0.05 \mathrm{D}$ and the $L=1.0 \mathrm{D}$ cases at azimuthal angles of $120^{\circ}$ and $180^{\circ}$.

\section{Free-run PIV measurement results}

As mentioned above, free-run PIV measurements were conducted in the present study to understand how the rotor-to-rotor interactions affect the aerodynamic and aeroacoustic performances of small UAVs. Figure 6 shows the measured free-run PIV results in terms of ensemble-averaged flow velocity and normalized in-plane turbulence kinetic energy (TKE, $0.5\left(\overline{u^{\prime 2}}+\overline{w^{2}}\right) / U_{t i p}^{2}$ ) for the single and twin-rotor cases, where the rotor tip speed (i.e., $U_{t i p}=\omega D / 2$ ) is $61 \mathrm{~m} / \mathrm{s}$. As shown quantitatively in Fig. 6 (a), the twin-rotor case presents a similar velocity distribution with that of the single rotor case. This explains the negligible effect of separation distance on measured mean thrusts as indicated in Fig. 3(a). Interestingly, as the induced flow convected downstream, the high-velocity region was found to converge from around the blade tip location (i.e., $\sim 0.4 D$ ) to near the root position (i.e., $\sim 0.2 D$ ), leading to the significant radial contraction behind the rotors. Though the global features of the velocity distribution behind the rotors were similar for both cases, some differences can still be identified from the velocity contours. Compared to the single rotor case, the measured velocity field in the twin-rotor case was slightly 'dragged' toward the adjacent rotor, which is believed to be caused by the Coanda effect (i.e., a phenomenon in which a flow tends to attract to a nearby object). As a result, the $W$ velocity component behind the twin-rotor was slightly lower than that of the single rotor in the corresponding down-part region $(-0.5<X / D<-0.2)$.

Fig. 6 (b) shows the comparisons of the normalized in-plane TKE distributions between the single and twin-rotor cases. Clearly, for the single rotor case, regions near the rotor tips were characterized with elevated in-plain TKE, which was caused by the periodic vortex shedding from the blade tip. A similar phenomenon can be observed for the twin-rotor case, except the region with enhanced in-plain TKE was found to be approximately doubled in the lateral direction, but 
reduced by half in the streamwise direction compared to the single rotor. Since the two rotors were only $0.05 D$ apart, the tip vortexes from rotors would interact severely with the nearby vortices, rendering enhanced in-plane TKE distributions in contrast to the single rotor case in the near rotor region. However, because of the intensive interaction within rotors, the associated coherent structures behind the twin-rotor would also dissipate much faster than that of the single rotor, therefore the region with the augmented in-plain TKE level would reduce rapidly as flow convected downstream. The elevated TKE level is believed to be not only related to the significant enhancement of thrust fluctuations discussed in the twin-rotor case (i.e., shown in Fig. 3), but also leaded to the augmentation of broadband noise (i.e., shown in Fig. 5), which will discuss further in the following stereoscopic PIV results.
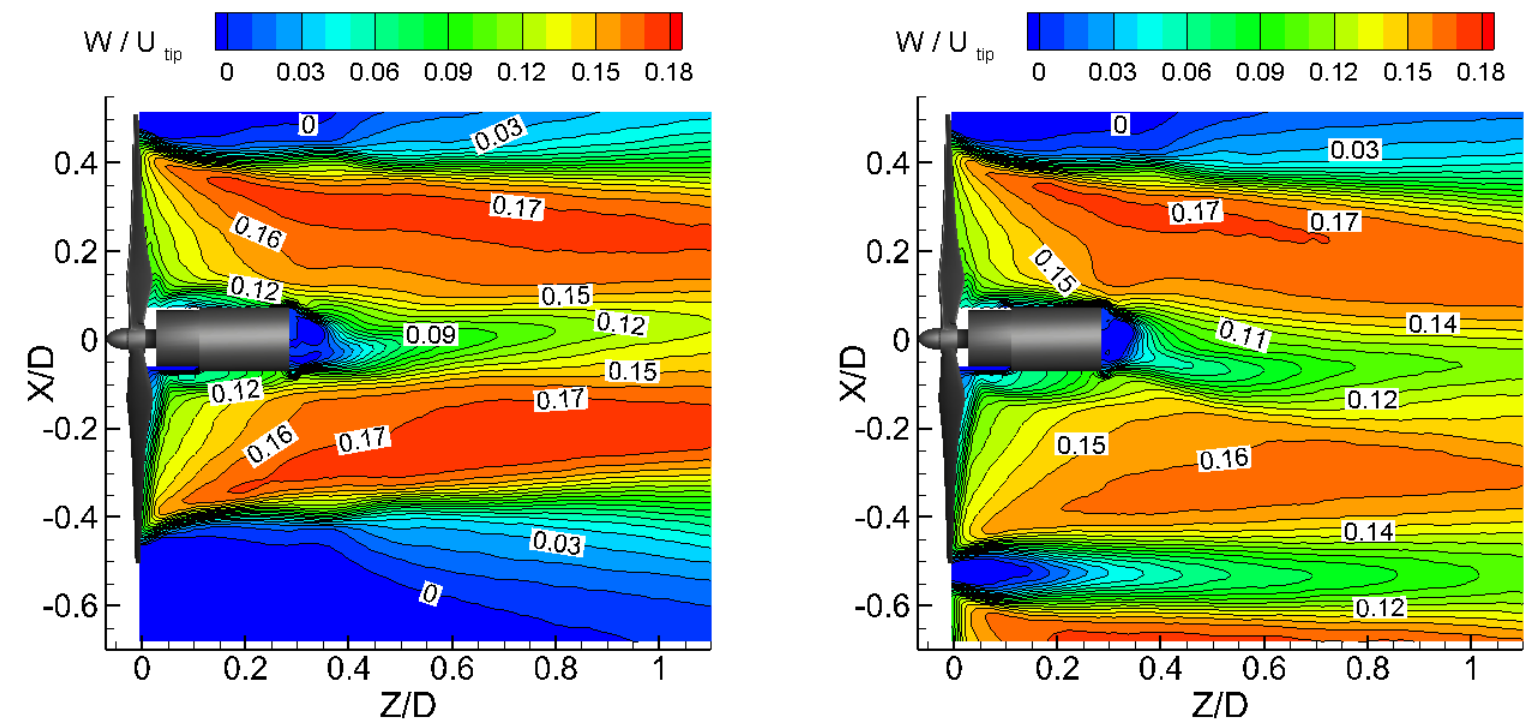

(a) Ensemble-averaged velocity field
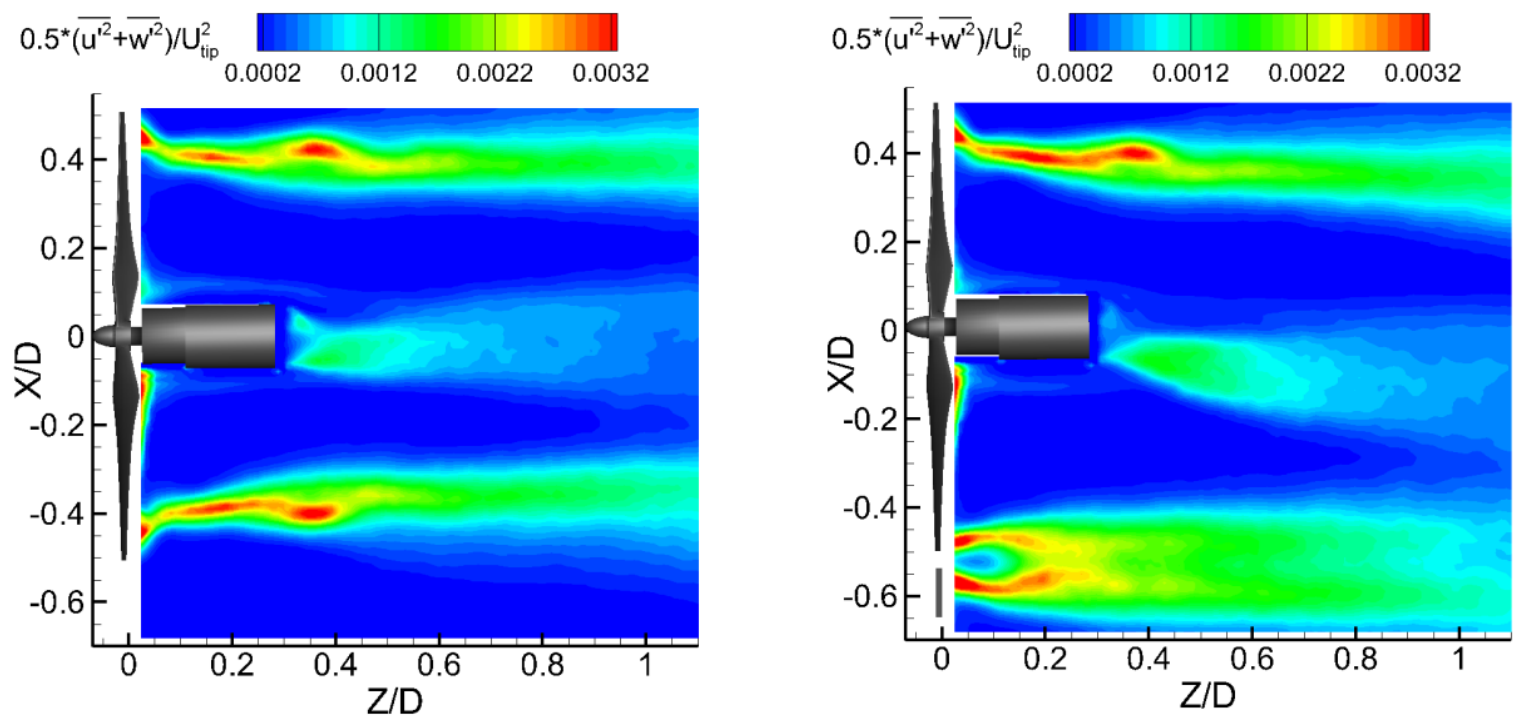

(b) Normalized in-plane TKE distribution

Figure 6. Measured PIV results for the single rotor (left) and twin-rotor (right, $L=0.05 D$ ) cases: (a) Ensemble-averaged velocity field, (b) Normalized in-plane TKE distribution. 


\section{Phase-locked PIV measurement results}

In the present study, phase-locked PIV measurements were also employed to produce "frozen" images of the unsteady vortex structures behind the rotor at different phase angles. Note that only the phase of left-hand rotor (i.e., shown in Fig. 2(a)) in the twin-rotor system was monitored during the phase-locked PIV test, whereas the other rotor can be treated as a free-run condition. Figure 7 shows the comparison of phase-averaged velocity distributions between the single and twin-rotor cases at two different phase angles (i.e., $\theta=0^{\circ}, 120^{\circ}$ ). During the experiment, the phase angle is defined as the angle between the vertical $Y-Z$ plane and the position of a pre-marked rotor blade. The pre-marked blade was in the most upward position (i.e., within the vertical $Y-Z$ plane) for the phase angle of $\theta=0^{\circ}$. As shown clearly in Fig. 7, the existence of wave-shaped flow structures can be observed clearly at the tip height for the single rotor case, which is closely related to the periodical shedding of tip vortices behind the rotor. The wave-shaped structures would propagate downstream as the phase angle increased. However, due to the rotor interactions for the twin-rotor case, the wave-shaped structures were found to dissipate much faster and become less pronounced in comparison with that of the single rotor case. In addition, these flow structures behind rotors were found to shift radially downward to the adjacent rotor, which is consistent with the measured results shown in Fig. 6.
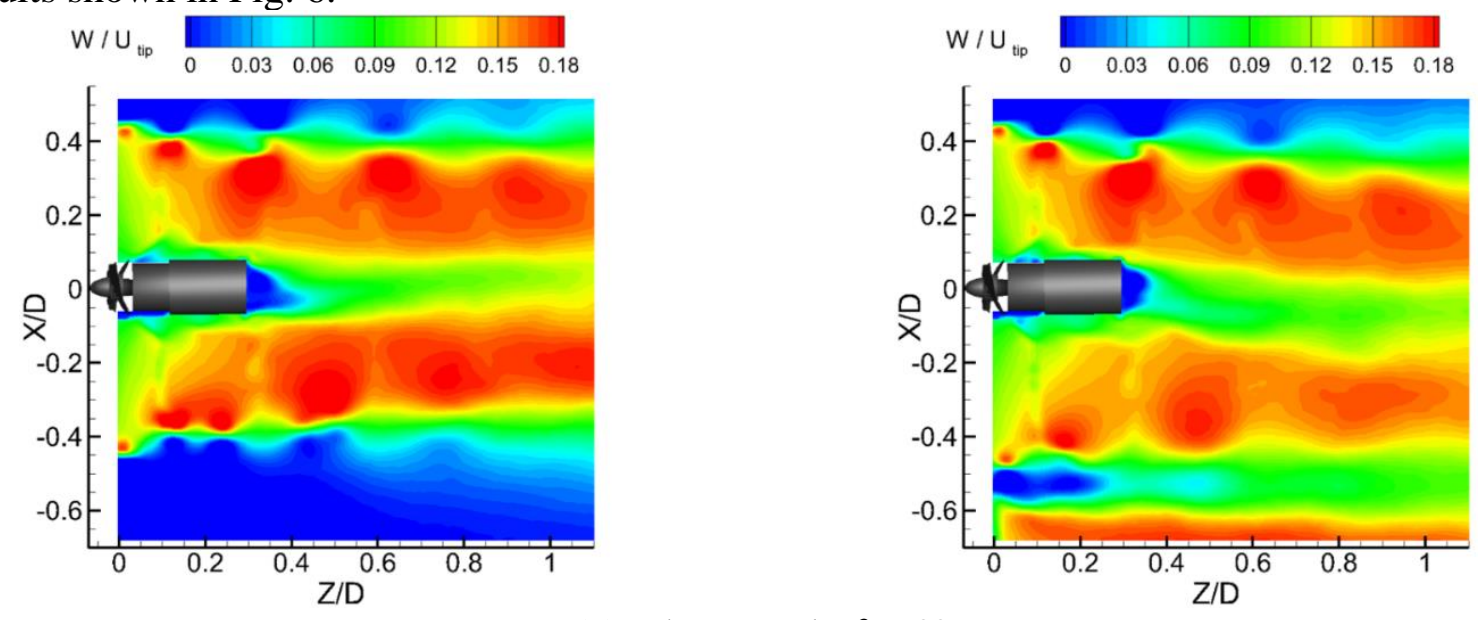

(a). Phase angle $\theta=0^{\circ}$
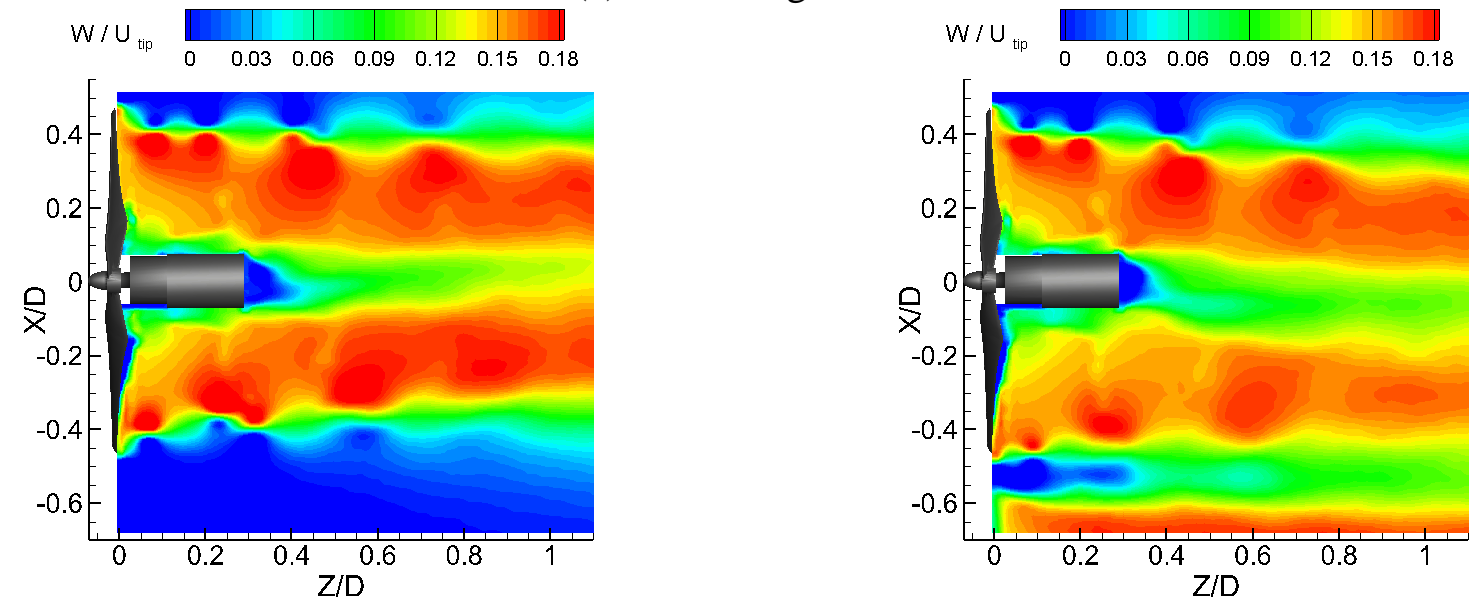

(a). Phase angle $\theta=0^{\circ}$

Figure 7. Phase-locked flow velocity distributions for the single rotor (left) and twin-rotor (right, $L=0.05 D$ ) configurations 
Figure 8 shows the comparison of the phase-locked vorticity distributions between the single rotor and twin-rotor cases, which can be used to reveal the effect of rotor interactions on the unsteady vortices more clearly. Here, the separation distance is $0.05 D$. As expected, the general features of the vorticity distributions were found to be quite similar for both cases, especially for the upper-part region (i.e., as indicated using red dash line). Flows behind the rotors were distinguished by pairs of tip vortices (i.e., \# 1 - 5) and shear layers (i.e., \# a - d) that trailed after the blades. Note that, the tip vortices were formed because of the pressure differences within the two sides of blade, while the shear layers were generated due to the merging of boundary layers from the upper and lower surfaces of the blade.
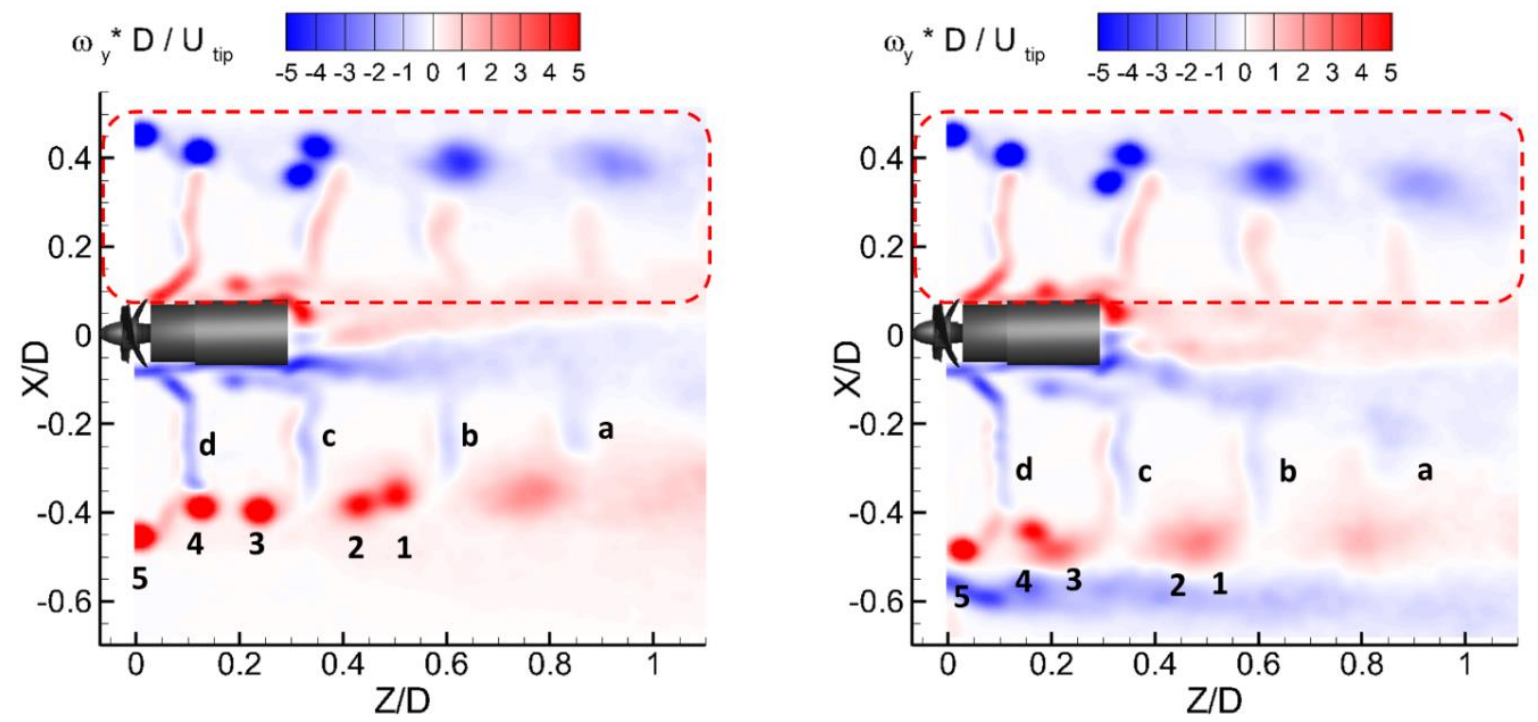

(a) Phase angle $\theta=0^{\circ}$.
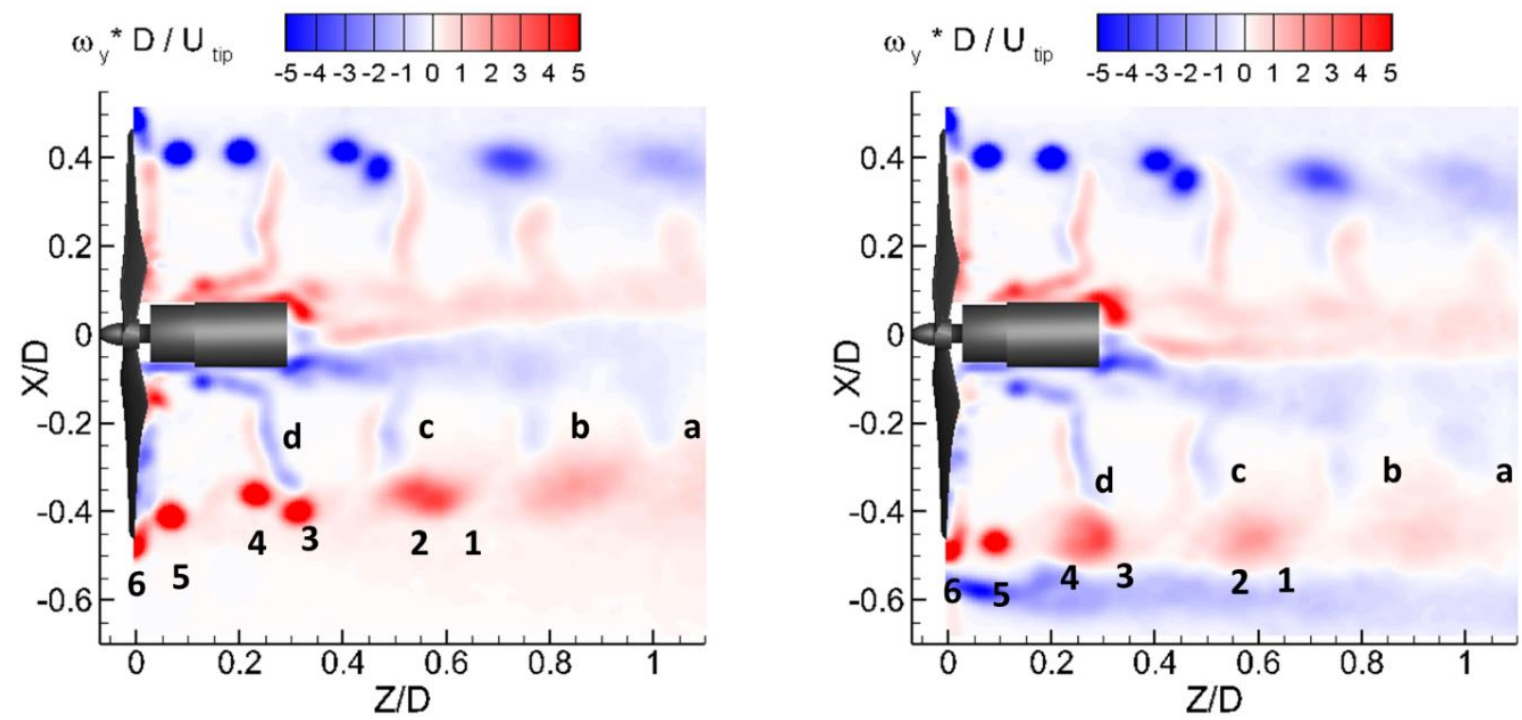

(b) Phase angle $\theta=120^{\circ}$

Figure 8. Phase-locked vorticity distributions for the single rotor (left) and twin rotors (right, $L=0.05 D$ ) configurations: 
For the single rotor case, when the pre-marked blade rotated over the PIV measurement plane, a tip vortex would shed from the tip and propagate axially downstream, shown in Fig. 8 (a). During this process, pairs of vortex structures (i.e., such as vortex \# $\mathbf{4}$ with \# $\mathbf{3}$, and vortex \# $\mathbf{2}$ with \# 1) would slowly merge into an integral one at $\sim 0.7 \mathrm{D}$. As the flow convected further downstream, they would completely vanish beyond $1.0 D$ due to the aperiodic and highly turbulent flow in the rotor wake. As for the twin-rotor case (i.e., $L=0.05 D$ ), though similar distributions can be observed for the shear layer (i.e., \# a - d), tip vortices were found to merge and dissipate much faster in comparison with that of the single rotor case. Representative examples are the isolated vortices (i.e., \# 1, \# 2, \# 3, \# 4), shown in the case of single rotor, that become much harder to discern as $Z / D>0.2$ in twin-rotor case.

As the phase angle increased to $\theta=120^{\circ}$, the associated turbulent structures, such as the shear layers and tip vortices, were found to propagate downstream in contrast to that of $\theta=0^{\circ}$. Focusing on the evolution of the vortex sheet \# $\mathbf{d}$ (i.e., shear layer \# d), it was initially adjacent to tip vortex \# 4. As vortices convected axially downstream, however the outboard edge of the shear layer \# $\mathbf{d}$ in Fig. 8 (b) was found to interact with the tip vortex \# $\mathbf{3}$ rather than vortex \# $\mathbf{4}$. This is because the convecting speed (i.e., $W$ velocity shown in Fig. 7) of the shear layer was much higher than that of the tip vortex, thus the outboard edge of the shear layer would surpass the corresponding vortex, leading to complex vortex interactions. Very similar phenomena were also reported by Leishman (Leishman 2006), who described the wake characteristics behind a helicopter rotor.

\section{E. Stereoscopic PIV measurement results}

To further explore the underlying physics pertinent to rotor-to-rotor interactions of small UAVs, a stereoscopic PIV (SPIV) system was also utilized to quantify the complex flow field behind the rotors at multiple locations along the induced flow direction. Note that the vectors shown in Figure 9 and 10 are the resultant vectors of in-plane radial and tangential velocities behind the rotor.

Fig. 9 and Fig. 10 show the comparisons of the ensemble-averaged turbulent quantities between the single rotor and twin-rotor cases at the downstream locations of $X / D=0.1$ and 1.0 respectively. As revealed clearly in Fig. 9 (a), the mean velocity contour (i.e., $W$ component) behind the single rotor was found to be circular and symmetric as expected. Interestingly, the rotating direction of the wake flow was found to be in the same direction with that of the rotor blade (i.e., both in counter-clockwise), which is contrary to the wind turbine scenario (Hu et al. 2016; Wang et al. 2016), where the swirling direction of the wake is opposite to the wind turbine rotation. This phenomenon is caused by an inherent working mechanism difference where the rotor drives the flow in the UAV case, but the it is the flow that drives the rotor in the wind turbine case. It should also be noted that while the velocity distribution behind the single rotor was in a circular shape, a droplet-shaped velocity field was observed for the twin-rotor case due to the flow disturbance from the nearby rotor. Carefully inspecting the flow features behind the twin rotors, a region with flow separation was identified at the top-right corner, which is believed to be the resultant effect of upwash flow and radial flow. Within the interaction region, the two rotors would generate a steady upwash flow that interacts severely with the radial flow, resulting in the flow separation shown in Fig. 9 (a).

As the airflow traveled further downstream to the $X / D=1.0$ plane, illustrated in Fig. 10 (a), the previous circular-shaped velocity field behind the single rotor was transformed into a 'horseshoe' shape. Not only that but also the orientation of the velocity contour was found to rotate 
by $\sim 35^{\circ}$ from its original position (i.e., in $X / D=0.1$ plane). Due to the flow inertia, the air stream would continue rotating after being accelerated by the blade as it traveled further downstream. Though a similar deviation angle was observed for the twin-rotor case, the velocity contour was found to bend slightly toward the adjacent rotor in comparison with that of the baseline, especially for the region close to the flow separation region (i.e., region in black dash line). This phenomenon is believed to be closely related to the aforementioned Coanda effect (i.e., Fig. 6) where the induced airflow behind the twin rotors would be attracted and bent toward the nearby rotor.

Fig. 9 (b) shows the measured normalized TKE distributions $\left(0.5\left(\overline{u^{\prime 2}}+\overline{v^{\prime 2}}+\overline{w^{\prime 2}}\right) / U_{\text {tip }}^{2}\right)$ for the single rotor case in comparison with that of the twin-rotor case in the $X / D=0.1$ plane. Clearly, an O-ring shaped TKE distribution was found to be the dominant feature for the single rotor case, which is because of the periodic vortex shedding from the rotor tip. The measured results are in accordance with the measured planar PIV results shown in Fig. 6.

Though the general pattern was found to be similar for both cases, except a region with significantly high TKE levels was observed at the top-right corner for the twin-rotor case. As expected, flow separation would lead to greatly enhanced turbulence fluctuations (i.e., TKE), which consequently resulted in dramatically intensified thrust fluctuations for the twin-rotor case as mentioned in Fig. 3 (b). This also confirmed the conjecture that the enhanced broadband noise shown in Fig. 5 was caused by the augmented TKE level. Imagine that as the blade struck through the separation region, it would certainly suffer strong fluctuations, leading to augmented TKE and broadband noise. Due to the viscous dissipation within the shear layer, the measured TKE levels for both cases were found to decrease rapidly and reach a similar level at the location of $X / D=1.0$ (i.e., shown in Fig. 10 (b)). Therefore, high TKE zone could only exist in the near-blade region since it dissipates rapidly as induced flow propagated downstream.

Figure 11 shows the evolution of the ensemble-averaged streamwise velocity and the normalized TKE distributions for the single and twin-rotor cases at three typical streamwise locations (i.e., $X / D=0.1,0.5$, and 1.0). As shown clearly in the figure, the mean velocity fields for both cases were found to become more uniform as induced flow convected downstream. In addition, the orientation of the induced flows was found to rotate by approximately $35^{\circ}$ (i.e., in the plane of $X / D=0.1$ ) in comparison with its original vertical position (i.e., in the plane of $X / D=$ 1.0). As for the normalized TKE distributions given in Fig. 11 (b), a significantly higher TKE level was initially observed at the top-right region of twin-rotor case in $X / D=0.1$ plane, but it then decreased rapidly and reached a similar level with that of the single rotor due to the viscous dissipation within the shear layer.

Based on above measured results, the twin-rotor case with $L / D=0.05$ experienced the strongest flow interactions within the rotors. Adding a wingtip, rather than enlarging the separation distance, might be a practical method to alleviate the flow interaction effects if UAV designers aren't willing to sacrifice the compactness of the drones. 

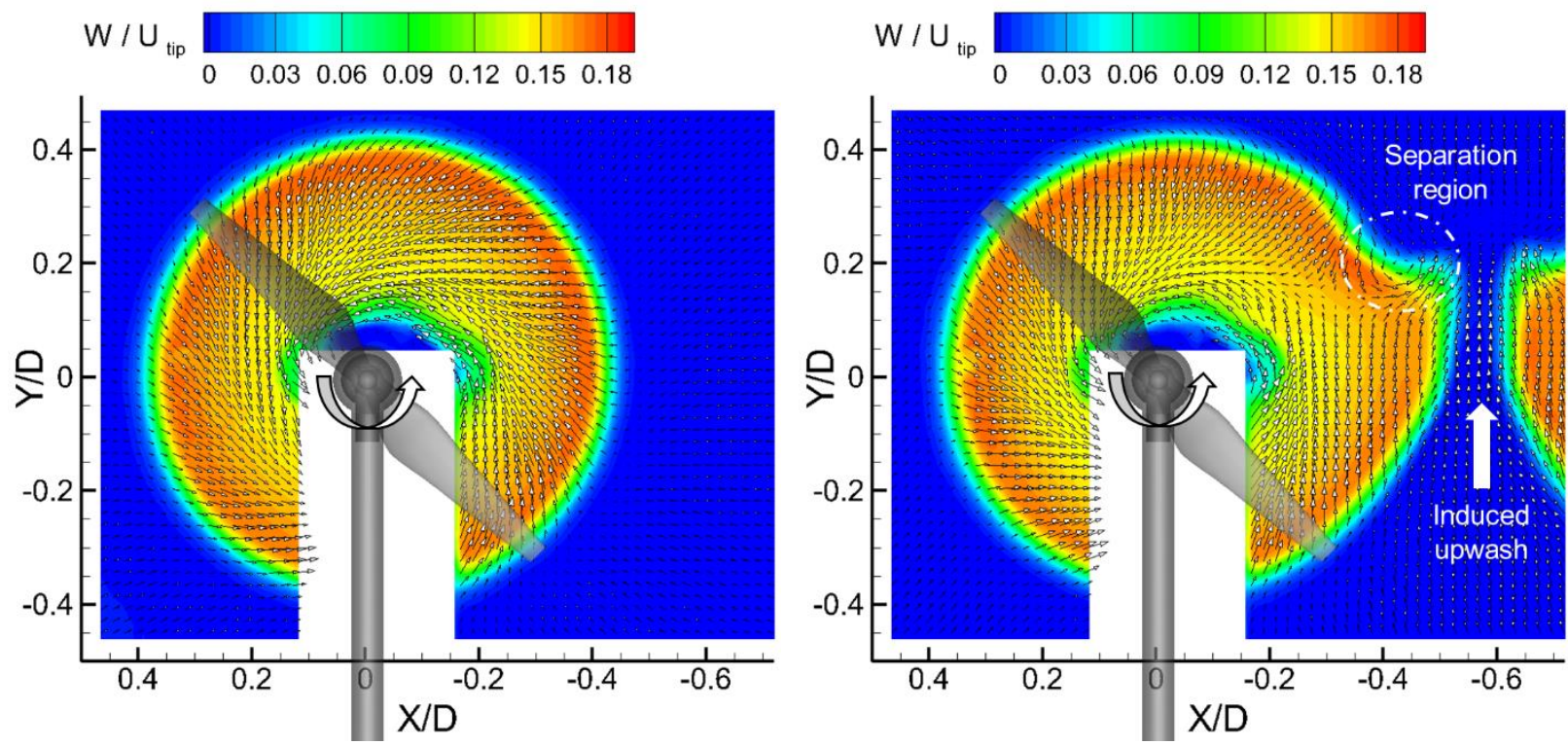

(a) Ensemble-averaged velocity field
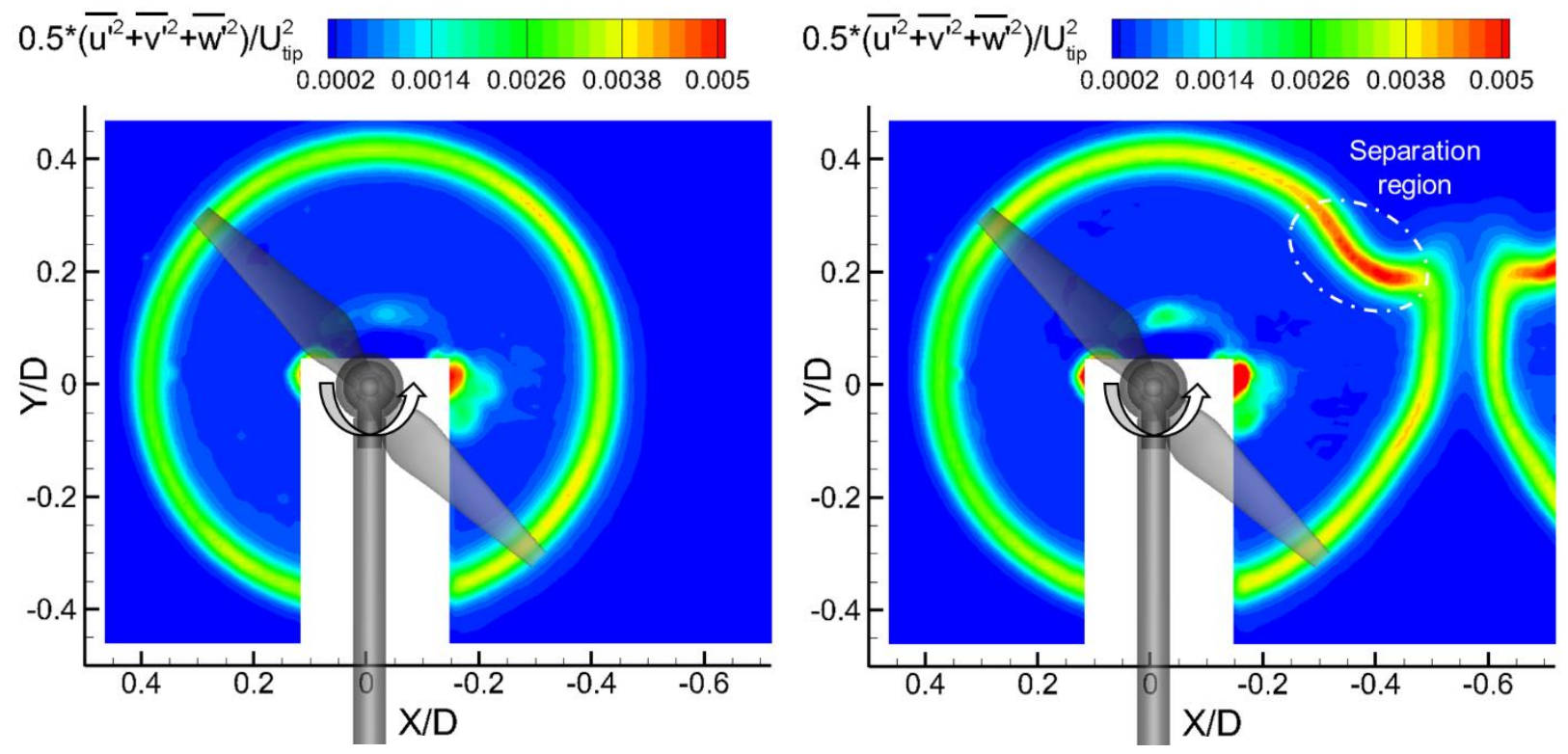

(b) Normalized TKE distribution

Figure 9. Measured SPIV results for the single rotor (left) and twin-rotor (right, $L=0.05 D$ ) configurations in the $X / D=0.1$ cross plane. 

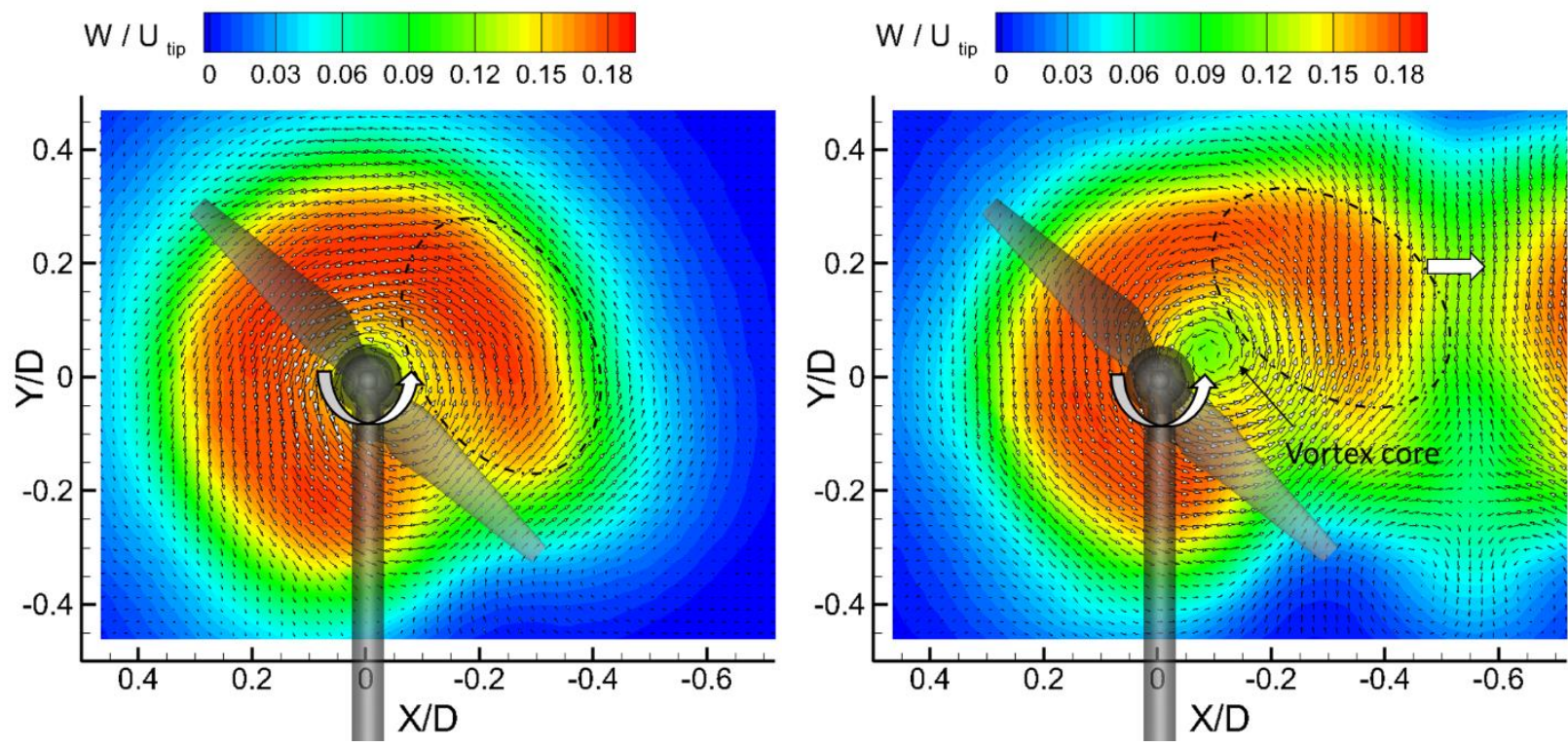

(a) (a) Ensemble-averaged velocity field

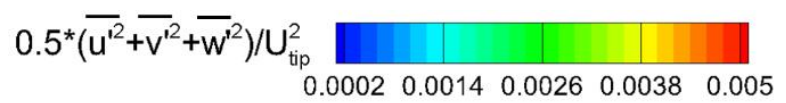
$0.5^{\star}\left(\overline{u^{\prime 2}}+\overline{v^{\prime 2}}+\overline{w^{\prime 2}}\right) / U_{\text {tip }}^{2}$
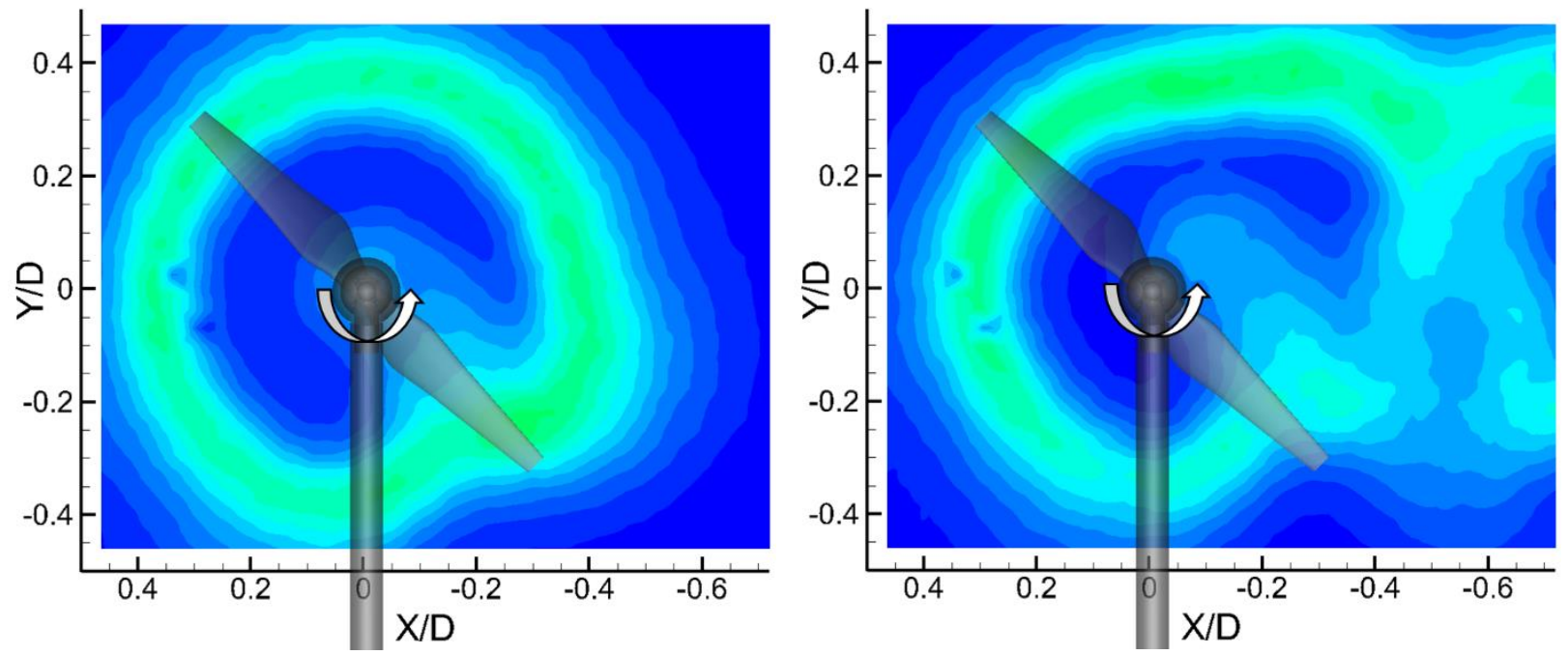

(b) Normalized TKE distribution

Figure 10. Stereoscopic PIV results for the single rotor (left) and twin-rotor (right, $L=0.05 D$ ) configurations in the $X / D=1.0$ cross plane 

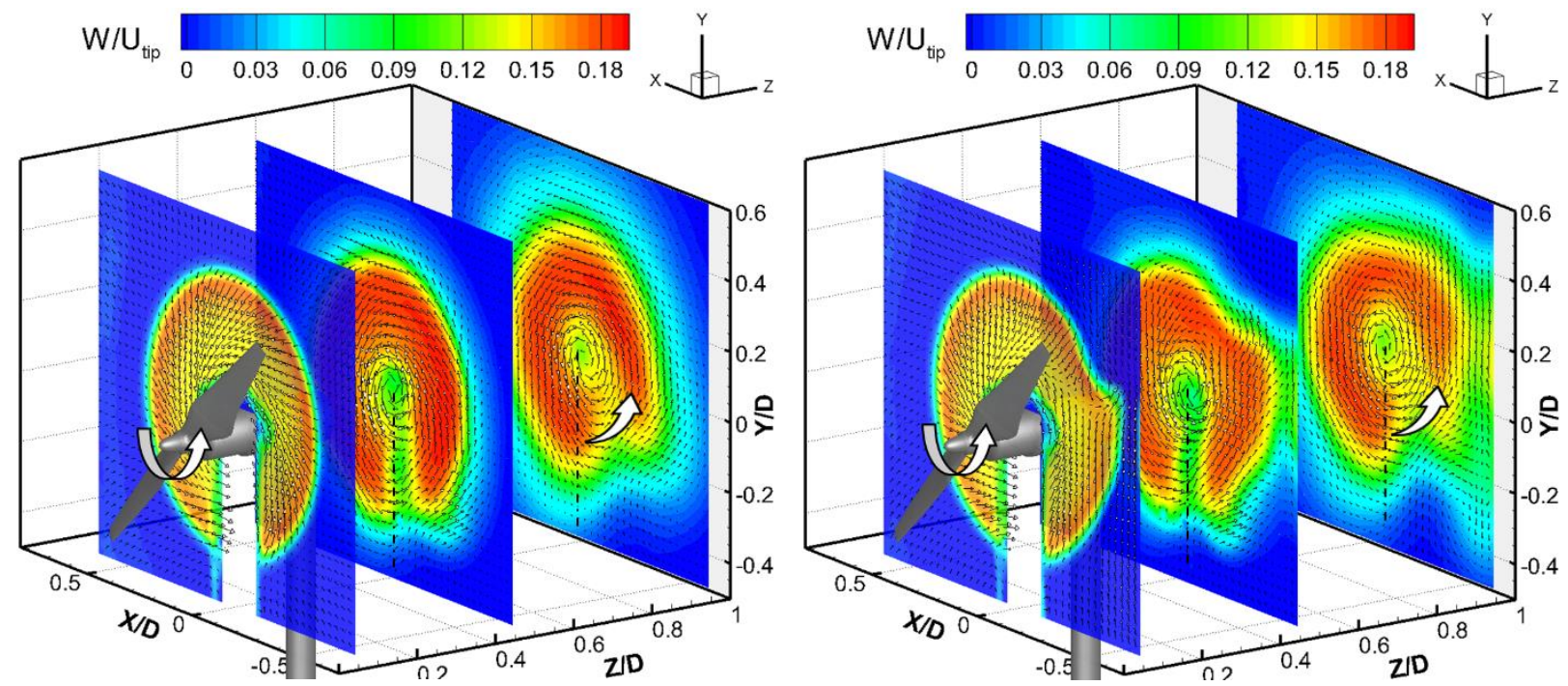

(a) Ensemble-averaged velocity field,
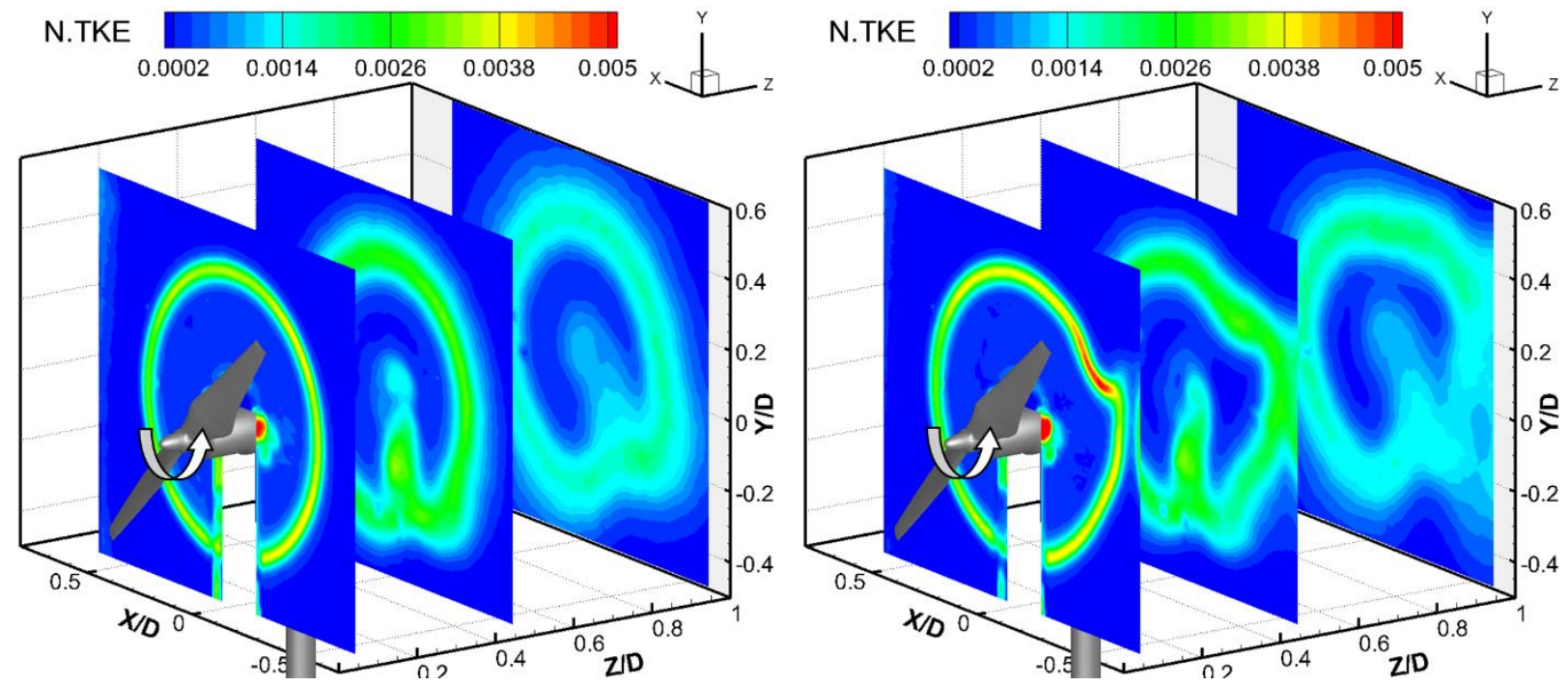

b) Normalized TKE distribution.

Figure 11. Stereoscopic PIV results for the single rotor (left) and twin rotors (right, $L=0.05 D$ ) configurations at $X / D=0.1,0.5$, and 1.0 locations.

\section{Conclusions}

An experimental investigation was performed to study rotor-to-rotor interactions on the aerodynamic and aeroacoustic performances of small UAVs. While the JR3 force transducer and microphone were used to quantify the thrust and noise levels of the rotors, a high-resolution Particle Image Velocimetry (PIV) system was used to conduct detailed flow field measurements to reveal the dynamic interactions between the rotors. The effects of separation distance $(L=$ $0.05 D, 0.1 D, 0.2 D$, and $1.0 D$ ) on the aerodynamic and aeroacoustic performance of UAVs were evaluated in detail based on the quantitative force, noise, and PIV measurements. 
It was found that, while the separation distance had a negligible effect on the thrust coefficient of the rotor (i.e., variation within $2 \%$ for all test cases), the thrust fluctuations (i.e., thrust standard deviation) were found to increase dramatically as the separation distance became smaller. More specifically, the measured thrust fluctuations for the twin-rotor case (i.e., $L=0.05 D$ ) were found to be $\sim 2.4$ times larger than that of the baseline case. This is believed to be caused by the complex flow interactions within rotors as revealed by the detailed PIV measurements. It was also found that the noise distribution for the twin-rotor case is a function of both azimuthal angle and separation distance, where the measured noise was found to increase as the azimuthal angle increased from $90^{\circ}$ (i.e., side position) to $180^{\circ}$ (i.e., right ahead the rotors), and to increase as the separation distance reduced from $L=1.0 D$ to $L=0.05 D$. A maximum noise enhancement of $\sim 3$ $\mathrm{dB}$ was recorded for the $L=0.05 \mathrm{D}$ case in comparison to that of the $L=1.0 \mathrm{D}$ case, which is the result of both tonal and broadband noise augmentations as indicated in the sound spectrum analysis.

As shown quantitatively from the planar PIV and Stereoscopic PIV measurement results, the induced flow behind the rotors was found to contract radially toward the axis of the rotor as the flow convected downstream. For the single rotor case, while most of the regions in the wake were devoid of flow structures, only the region near the blade tips was characterized by elevated inplain TKE (i.e., in $X-Z$ plane) due to periodic tip vortex shedding. A similar phenomenon was observed for the twin-rotor case, except that the TKE region was significantly higher at the topright area than that of single rotor case. Due to the resultant effect of upwash and radial flows in the near wake of the twin-rotor case, a region with a flow separation was identified in the $X$ - $Y$ cross plane, which led to significantly higher TKE distributions and thrust fluctuations behind the twin rotors. It should also be noted that the velocity field for the twin-rotor case was found to be attracted and bent toward the nearby rotor due to the Coanda effect.

In general, the measured quantitative results given in the present study are believed to be very beneficial in understanding how rotor-rotor interactions affect the aerodynamic and aeroacoustic performances of small UAVs. It depicted a vivid picture regarding the complex flow features behind the rotors, which further explained their correlations with the enhanced force fluctuations and noise levels. Such quantitative information is highly desirable to elucidate the underlying physics pertinent to rotor interactions, and to explore/optimize design paradigms for better commercial UAV designs.

\section{Acknowledgments}

The supported of National Science Foundation (USF) USA with Grant numbers of CBET1438099 is gratefully acknowledged.

\section{References}

Blackstock DT (2000) Fundamentals of physical acoustics. A WILEY-INTERSCIENCE

Bouabdallah S, Becker M, Siegwart R (2007) Autonomous miniature flying robots: coming soon! - Research, Development, and Results. IEEE Robot Autom Mag 14:88-98. https://doi.org/10.1109/MRA.2007.901323

Brandt J, Selig M (2011) Propeller Performance Data at Low Reynolds Numbers. In: 49th AIAA Aerospace Sciences Meeting including the New Horizons Forum and Aerospace Exposition. American Institute of Aeronautics and Astronautics 
Bristeau P, Martin P, Salaün E (2009) The role of propeller aerodynamics in the model of a quadrotor UAV. In: Control Conference (ECC

Carolus T, Schneider M, Reese H (2007) Axial flow fan broad-band noise and prediction. J Sound Vib 300:50-70. https://doi.org/10.1016/j.jsv.2006.07.025

Ditmer MA, Vincent JB, Werden LK, et al (2015) Bears Show a Physiological but Limited Behavioral Response to Unmanned Aerial Vehicles. Curr Biol 25:2278-2283. https://doi.org/10.1016/j.cub.2015.07.024

Goebel ME, Perryman WL, Hinke JT, et al (2015) A small unmanned aerial system for estimating abundance and size of Antarctic predators. Polar Biol 38:619-630. https://doi.org/10.1007/s00300-014-1625-4

Gur O, Rosen A (2009) Design of Quiet Propeller for an Electric Mini Unmanned Air Vehicle. J Propuls Power 25:717-728. https://doi.org/10.2514/1.38814

Hu H, Wei T, Wang Z (2016) An Experimental Study on the Wake Characteristics of Dual-Rotor Wind Turbines by Using a Stereoscopic PIV Technique. 34th AIAA Appl Aerodyn Conf

Intaratep N, Alexander WN, Devenport WJ, et al (2016) Experimental Study of Quadcopter Acoustics and Performance at Static Thrust Conditions. In: 22nd AIAA/CEAS Aeroacoustics Conference. American Institute of Aeronautics and Astronautics, Reston, Virginia

Leishman G (2006) Principles of helicopter aerodynamics. Cambridge University Press

Leslie A, Wong KC, Auld D (2008) Broadband Noise Reduction on a mini-UAV Propeller. In: 14th AIAA/CEAS Aeroacoustics Conference (29th AIAA Aeroacoustics Conference). American Institute of Aeronautics and Astronautics, Reston, Virigina

Lucieer A, Turner D, King DH, Robinson SA (2014) Using an Unmanned Aerial Vehicle (UAV) to capture micro-topography of Antarctic moss beds. Int J Appl Earth Obs Geoinf 27:53-62. https://doi.org/10.1016/j.jag.2013.05.011

Merchant M, Miller LS (2006) Propeller Performance Measurement for Low Reynolds Number UAV Applications. In: 44th AIAA Aerospace Sciences Meeting and Exhibit. American Institute of Aeronautics and Astronautics, Reston, Virigina

Otsuka H, Nagatani K (2016) Thrust loss saving design of overlapping rotor arrangement on small multirotor unmanned aerial vehicles. In: 2016 IEEE International Conference on Robotics and Automation (ICRA). IEEE, pp 3242-3248

Russell C, Jung J, Willink G, Glasner B (2016) Wind Tunnel and Hover Performance Test Results for Multicopter UAS Vehicles. In: AHS 72nd Annual Forum. FL

Sinibaldi G, Marino L (2013) Experimental analysis on the noise of propellers for small UAV. Appl Acoust 74:79-88. https://doi.org/10.1016/j.apacoust.2012.06.011

Wang Z, Tian W, Ozbay A, et al (2016) An experimental study on the aeromechanics and wake characteristics of a novel twin-rotor wind turbine in a turbulent boundary layer flow. Exp Fluids 57:150. https://doi.org/10.1007/s00348-016-2233-6

Yoon S, Lee HCH, Pulliam TH (2016) Computational Analysis of Multi-Rotor Flows. In: 54th AIAA Aerospace Sciences Meeting. American Institute of Aeronautics and Astronautics, Reston, Virginia 